\title{
Automated Interpretation of Cardiac Scintigrams
}

\author{
Jens Richter ${ }^{2}$, Anders Ericsson ${ }^{1}$, Kalle $\AA$ ström ${ }^{1}$, Fredrik Kahl ${ }^{1}$, and \\ Lars Edenbrant ${ }^{3}$ \\ 1 Mathematics, Centre for Mathematical Sciences, \\ Lund Institute of Technology, Lund University, Sweden \\ 2 WeAidU, IDEON, Lund, Sweden \\ 3 Department of Clinical Physiology, \\ Lund University, Lund, Sweden
}

\begin{abstract}
The purpose of this study was to develop an automated method for the segmentation of the heart in a 3-D cardiac scintigram. This is immediately useful for eliminating a manual step in a previous version of a decision support system.

The automatic segmentation method uses a statistical 3D-model, inspired by Active Shape, which locates the base and apex automatically from a cardiac scintigram. Key features of this algorithm are that it can handle cases where there is no or very little activity in the apex and also if there are additional parts of the heart where there is little activity. The algorithm has been tested on approximately 2000 cardiac scintigrams.
\end{abstract}

\section{Introduction}

When a cardiac infarction is suspected, a cardiac scintigram is a common examination procedure. At a number of hospitals in Sweden a decision support system is used to analyze such images. The algorithm that is presented in this article is part of that system, which now is fully automatic. The system helps physicians with a second opinion in particular examinations.

A cardiac scintigram is a $3 \mathrm{D}$ voxel volume (3D image of size, $64 \times 64 \times 20$ pixels is typical) of a heart, see Figure 1.
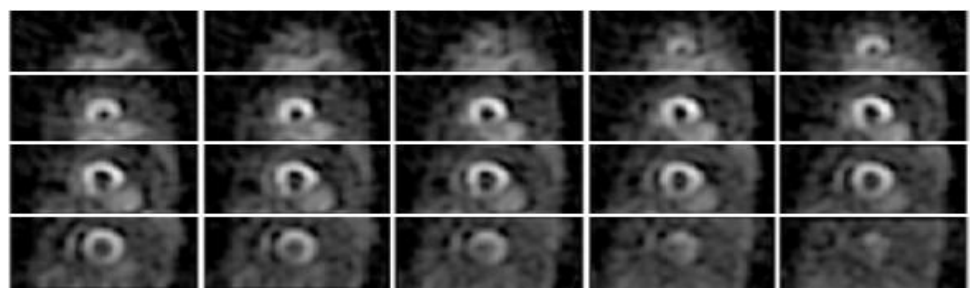

Fig. 1. 20 of 23 image slices of a typical cardiac scintigram. 
In this figure the voxel volume is illustrated as a number of $2 \mathrm{D}$-image slices, which is a common way to present cardiac scintigrams. In the previous version of the decision support system, the user manually had to select in which image slice the top (lat. base) of the heart was present and in which slice the bottom (lat. apex) was present. This is often difficult, especially if the condition of the heart in question is bad. Since this task is of a subjective nature, the result varied much among different physicians. A minor study of this problem made it clear, that the choice of in what slice the top and bottom is present in a scintigram, can vary as much as up to \pm 2 image slices. Thus, the reliability of the former system was questioned. A fully automatic system is not subjective in this sense and would therefore eliminate the error induced by this variability. The system would also be less interactive, less time-consuming, easier to use and thus more accepted among physicians.

Here we propose to use a statistical 3D-model of the cardiac to find the position and the shape of a previously unseen image. The method is described in Section 2. In Section 3, results and experimental validation are given.

\section{Method}

The theory is much inspired by the work on Active Shape [3]. The main differences are that the landmarks are located automatically and that the search algorithm is adapted to scintigrams. Therefore the focus lies on these issues.

\subsection{Automatic placement of landmarks}

Many attempts have been made to automate the process of locating corresponding landmarks. There have been many suggestions for $2 \mathrm{D}[1,2,7,8]$. Local geometric properties, such as geodesics, have been tested for surfaces [9]. In [5, 6] the description length of the model is minimised to establish correspondences even in 3D. This algorithm has shown to be successful, but it is too computationally expensive for our purposes. Here we suggest another method for locating landmarks automatically.

A fairly geometrically simple model of the heart is presented in Figure 2. It is a joined elliptic paraboloid and a cylinder. The boundary joining the elliptic paraboloid and the cylinder we call the top-ring. Due to the nature of cardiac scintigrams, landmarks are defined in one way on the cylinder and in another way on the elliptic paraboloid. However, both rely on that the top-ring is located. The landmarks position can easiest be illustrated using the simple model of the heart, see Figure 2.

Locating the top-ring. Starting with the uppermost image slice of a scintigram, intensity peaks are searched for along the $k$ rays that coincide in the point $P^{\prime}$ of the image slice, see Figure 2. The point $P^{\prime}$ is the $\mathrm{x}$ and y coordinates of $P$ ( $P$ is defined below). The line search is analogous to that in $3 \mathrm{D}$, which is described in the next paragraph. 

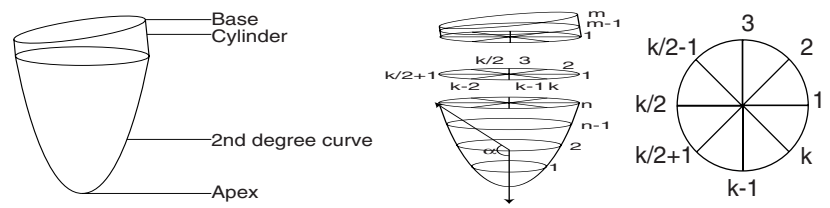

Fig. 2. To the left, our simple model of the heart. In the middle, the definition of landmarks. To the right, searching for the top-ring.

The first tolerable intensity peak at a reasonable distance from the center corresponds to the wall of the heart. If the wall has not been located in all $k$ search directions, continue searching the next image slice.

Definition of landmarks on the elliptic paraboloid. See Figure 2, nk landmarks are defined on the elliptic paraboloid. The figure is schematic and the landmarks will in general not lie on the elliptic paraboloid surface. Let $P$ be the center of gravity of the heart that has been masked out. The top ring and $P$ defines the angle $\alpha$. Imaging a cone with top angle $i \alpha / n(i=1, \ldots, n)$ at $P$. Place $k$ rays equally distributed on the i:th cone through $P$. Varying $i$ from 1 to $n$, gives a total of $n k$ rays through $P$. The strongest edges within a distance of 10 voxels from $P$ along these rays defines the landmarks.

To be able to determine the position of the edges with sub pixel precision along a ray, the image is interpolated with ideal interpolation followed by Gaussian smoothing. This is approximately the same as using the Gaussian

$$
h(x, y, z)=\frac{1}{\sigma^{1 / 2}(2 \pi)^{3 / 2}} \exp \left(\frac{x^{2}+y^{2}+z^{2}}{2 \sigma^{2}}\right)
$$

as interpolation function. The continuous image $I_{c}$ is calculated from the discrete image $I_{d}$ according to

$$
I_{c}(x, y, z)=\sum_{i, j, k \in \mathcal{Z}^{3}} h(x \quad i, y \quad j, z \quad k) I_{d}(i, j, k) .
$$

The function $g(t)=I_{c}(\mathbf{x}+t \mathbf{n})$ is the intensity along a line $\mathbf{x}+t \mathbf{n}$

$$
\begin{aligned}
g^{\prime}(t) & =\mathbf{n}^{T} \nabla I_{c}(\mathbf{x}+t \mathbf{n}) \\
& =\mathbf{n}^{T} \sum_{i, j, k \in \mathcal{Z}^{3}} \nabla h(x \quad i, y \quad j, z \quad k) I_{d}(i, j, k) .
\end{aligned}
$$

The extreme points of $g(t)$ is located using Newton Raphson's method.

Definition of landmarks on the cylinder From the landmarks defined on the top-ring the edge is tracked in the $\mathrm{z}$-direction. Once the edge is too weak the search is stopped and $k$ paths have been found. Along each path $m$ landmarks are equally placed given a total of $m k$ landmarks on the cylinder. 


\subsection{Training an Active Shape Model}

Using the method described above, a number of landmarks, $(m+n) k$, in cardiac scintigrams from healthy patients can be located automatically. Once the landmarks have been located, the theory of Active Shape in 3D [4] can be applied straight ahead. This gives a statistical 3D-model of a cardiac, which will be used to locate the heart in a previously unseen scintigram.

\subsection{Fitting the 3D shape model to previously unseen images}

First an overview of the fitting algorithm is presented.

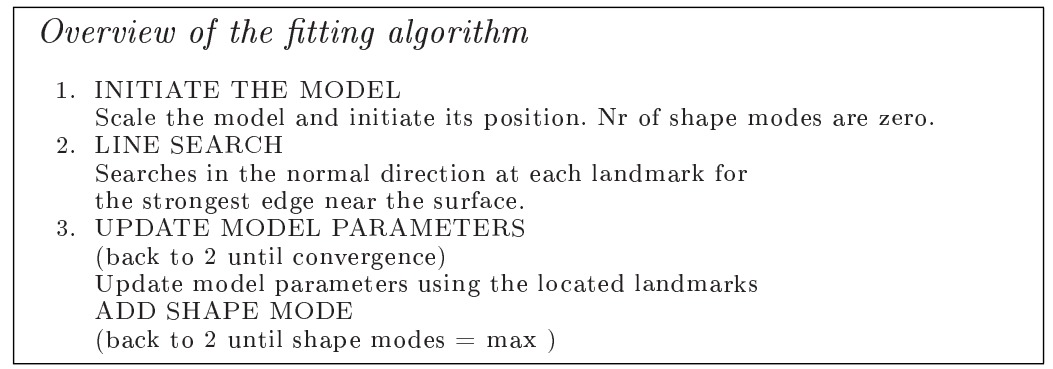

Algorithm 1 : Algorithm to fit the model of a heart to a cardiac scintigram.

1) Initiate the model. To initiate the model the top-ring and the bottom of the heart is located. This is done analogously as for the training set in 2.1. Since it is not certain that it locates all directions, the top-ring is set to be the slice where most directions are present. The bottom is the slice where least number of directions is located within a number of slices from the top-ring. The model is rescaled so that the top-ring and the bottom of the model corresponds to the image.

First the number of shape modes are set to zero. This means that it only translates and rescales the model in the first iteration.

2) Line search. At each landmark the normal is defined from its neighbors. A line search analogously to that described in 2.1 locates the nearest surrounding strongest edge. If an edge is located the coordinate of that landmark is updated. If no edge is located that landmark will be excluded from the calculation of the new model parameters.

3) Update model parameters. Applying Active Shape [4] the shape $\mathbf{x}$ is described as

$$
\left[\begin{array}{c}
x_{1} \\
\cdot \\
x_{i} \\
\cdot \\
x_{m}
\end{array}\right]=\left[\begin{array}{c}
\overline{x_{1}} \\
\cdot \\
\overline{x_{i}} \\
\cdot \\
\overline{x_{m}}
\end{array}\right]+\left[\begin{array}{ccc}
\phi_{11} & \cdots & \phi_{1 n} \\
\cdot & \cdot & \cdot \\
\phi_{i 1} & \cdots & \phi_{i n} \\
\cdot & \cdot & \cdot \\
\phi_{m 1} & \cdots & \phi_{m n}
\end{array}\right]\left[\begin{array}{c}
b_{1} \\
\vdots \\
b_{n}
\end{array}\right],
$$


where $\overline{\mathbf{x}}$ is the mean shape. $\boldsymbol{\Phi}$ is a $m \times n$ matrix with orthogonal columns containing the $n$ number of $m \times 1$ vectors of variation. The shape parameters $\mathbf{b}$ can be resolved by

$$
\mathbf{b}=\Phi^{\mathbf{T}}(\mathbf{x} \quad \overline{\mathbf{x}})
$$

Let $x_{i}$ be a missing landmark, corresponding to an edge that was not found in the second step sbove. Excluding the i:th row in (1), b can still be resolved as in (2), if only $m \geq n \quad$. If there are p missing landmarks, $\mathbf{b}$ can still be resolved if $m \geq n \quad p$. Putting $\mathbf{b}$ into (1) the shape $\mathbf{x}$ is retrieved. The missing landmarks can thus be estimated to be the most likely, given the non-missing landmarks.

\section{Results}
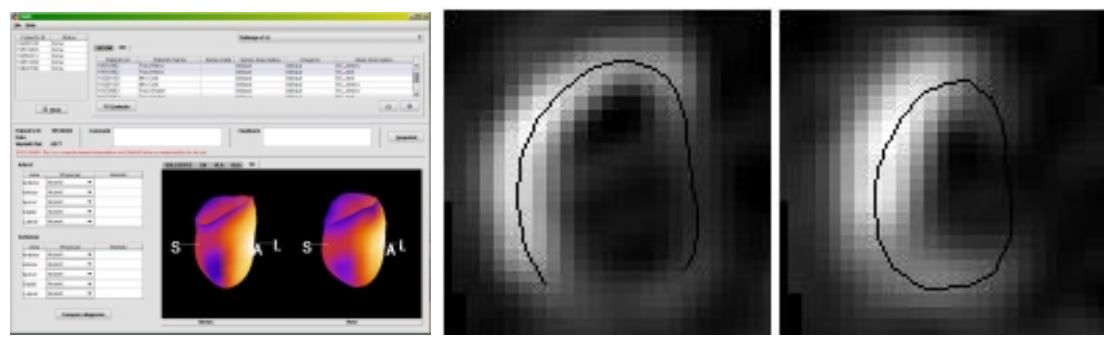

Fig. 3. To the left a snapshot of a new decision support system. To the right the slice number 12 and 16 of a cardiac scintigram are plotted together with its modelled heart.

The algorithm presented in this paper has been implemented in java and incorporated in the client version of a decision support system, see a snapshot of it in Figure 3. The snapshot shows the 3D visualization of the heart. A physician can rotate it in any angle and easily determine where the diseased area is (dark areas are diseased). The client version can be obtained at www . weaidu.com. The decision support system is currently used at approximately 15 hospitals in Sweden, Denmark, Finland, England and Italy. More than 2000 cardiac scintigrams have been analysed by the decision support system, including the active shape method. Two experts have studied approximately 500 of the 2000 cardiac scintigrams and They found that more than $99 \%$ of the cases were properly analysed by the new algorithm. The remaining cases have been automatically detected for manual processing.

\section{Discussion}

The purpose of this study was to develop an automated method for the segmentation of the heart in a cardiac scintigram. This is immediately useful for eliminating a manual step in a previous version of a decision support system. 
The segmentation problem is difficult because there can be large parts of the heart wall that are not visible in the cardiac scintigrams. The segmentation method needs to be able to handle this. In this paper we have shown how prior knowledge of heart shapes can be obtained and used to segment the heart.

In our next study we will more focus on what information that can be drawn out of the shape description. The shape and the condition of the heart are correlated.

\section{Acknowledgements}

This study was supported by grants from the Swedish Research Council (VR), project TFR 2000-221-606 and MFR 09893.

\section{References}

1. A. Baumberg and Hogg D. Learning flexible models from image sequences. In Proc. European Conf. on Computer Vision, ECCV'94, pages 299-308, 1994.

2. A. Benayoun, Ayache N., and Cohen I. Adaptive meshes and nonrigid motion computation. In Proc. International Conference on Pattern Recognition, ICPR'94, 1994.

3. T.F. Cootes, A. Hill, C.J Taylor, and Haslam J. Use of active shape models for locating structure in medical images. IEEE Trans. medical imaging, 12(6):355-365, 1994.

4. T.F Cootes and C.J. Taylor. Statistical Models of Appearance for Computer Vision. University of Manchester, 2001.

5. Rhodri H. Davies, Tim F. Cootes, John C. Waterton, and Chris J. Taylor. An efficient method for constructing optimal statistical shape models. In Medical Image Computing and Computer-Assisted Intervention MICCAI'囚001, pages 57-65, 2001.

6. Rhodri H. Davies, Carole J. Twining, Tim F. Cootes, John C. Waterton, and Chris J. Taylor. A minimum description length approach to statistical shape modeling. IEEE Trans. medical imaging, 21(5):525-537, 2002.

7. C. Kambhamettu and D.B. Goldgof. Points correspondences recovery in non-rigid motion. In Proc. Conf. Computer Vision and Pattern Recognition, CVPR'92, pages 222-237, 1992.

8. A. Kelemen, G. Szekely, and Gerig G. Elastic model-based segmentation of 3d neuroradiological data sets. IEEE Trans. medical imaging, 18(10):828-839, 1999.

9. Y. Wang, B.S. Peterson, and L.H Staib. Shape-based 3d surface correspondence using geodesics and local geometry. In Proc. Conf. Computer Vision and Pattern Recognition, CVPR'00, pages 644-651, 2000. 